\title{
Molecular Characterization of the Native (Non-Linked) CD160-HVEM Protein Complex Revealed by Initial Crystallographic Analysis
}

\author{
Simona Lenhartová ${ }^{1}$, Marek Nemčovič ${ }^{2}$, Radka Šebová ${ }^{1}$, Mário Benko ${ }^{1}$, Dirk M. Zajonc ${ }^{3,4}$ \\ and Ivana Nemčovičová $1, *$ (D) \\ 1 Biomedical Research Center, Slovak Academy of Sciences, Dúbravská cesta 9, 84505 Bratislava, Slovakia; \\ virusims@savba.sk (S.L.); virurama@savba.sk (R.Š.); virubenq@savba.sk (M.B.) \\ 2 Institute of Chemistry, Slovak Academy of Sciences, Dúbravská cesta 9, 84538 Bratislava, Slovakia; \\ chemnem@savba.sk \\ 3 Division of Immune Regulation, La Jolla Institute for Immunology, 9420 Athena Circle, \\ La Jolla, CA 92037, USA; dirk.zajonc@pfizer.com \\ 4 Cancer Immunology Discovery, Pfizer Inc., 10777 Science Center, San Diego, CA 92121, USA \\ * Correspondence: viruivka@savba.sk
}

Citation: Lenhartová, S.; Nemčovič, M.; Šebová, R.; Benko, M.; Zajonc, D.M.; Nemčovičová, I. Molecular Characterization of the Native (Non-Linked) CD160-HVEM Protein Complex Revealed by Initial Crystallographic Analysis. Crystals 2021, 11, 820. https://doi.org/ $10.3390 /$ cryst 11070820

Academic Editors: Ivana

Kutá Smatanová and Pavlína Maloy Řezáčová

Received: 1 July 2021

Accepted: 12 July 2021

Published: 15 July 2021

Publisher's Note: MDPI stays neutral with regard to jurisdictional claims in published maps and institutional affiliations.

Copyright: (c) 2021 by the authors. Licensee MDPI, Basel, Switzerland. This article is an open access article distributed under the terms and conditions of the Creative Commons Attribution (CC BY) license (https:// creativecommons.org/licenses/by/ $4.0 /)$.

\begin{abstract}
An increasing number of surface-exposed ligands and receptors acting on immune cells are being considered as a starting point in drug development applications. As they are dedicated to manipulating a wide range of immune responses, accurately predicting their molecular interactions will be necessary for the development of safe and effective therapeutics to enhance immune responses and vaccination. Here, we focused on the characterization of human CD160 and HVEM immune receptors, whose mutual engagement leads to bidirectional signaling (e.g., T cell inhibition, natural killer cell activation or mucosal immunity). In particular, our study reports on the molecule preparation, characterization and initial crystallographic analysis of the CD160-HVEM complex and both HVEM and CD160 in the absence of their binding partner. Despite the importance of the CD160-HVEM immune signaling and its therapeutic relevance, the structural and mechanistic basis underlying CD160-HVEM engagement has some controversial evidence. On one hand, there are studies reporting on the CD160 molecule in monomeric form that was produced by refolding from bacterial cells, or as a covalently linked single-chain complex with its ligand HVEM in insect cells. On the other hand, there are older reports providing evidence on the multimeric form of CD160 that acts directly on immune cells. In our study, the native non-linked CD160-HVEM complex was co-expressed in the baculovirus insect host, purified to homogeneity by anion-exchange chromatography to provide missing evidence of the trimeric form in solution. Its trimeric existence was also confirmed by the initial crystallographic analysis. The native CD160-HVEM complex crystallized in the orthorhombic space group with unit cell parameters that could accommodate one trimeric complex (3:3) in an asymmetric unit, thus providing ample space for the multimeric form. Crystals of the CD160-HVEM complex, CD160 trimer and HVEM monomer (reported in two space groups) diffracted to a minimum Bragg spacing of 2.8, 3.1 and 1.9/2.1 A resolution, respectively. The obtained data will lead to elucidating the native structure of the complex.
\end{abstract}

Keywords: CD160/BY55; HVEM/TNFRSF14/CD270; immune receptors; immunological synapse; $\mathrm{X}$-ray crystallography; receptor-ligand interactions

\section{Introduction}

The complexity of the ligand-receptor interactions of the immediate TNF (tumor necrosis factor) superfamily (SF) members engaging HVEM (herpes virus entry mediator; TNFRSF14) provides an unprecedented challenge in defining their mechanism of action and physiologic functions. Although the importance of the HVEM immune signaling 
and its therapeutic relevance are obvious, the structural and mechanistic basis underlying HVEM's engagement of CD160 has some controversial evidence. In some cases, CD160 is reported in monomeric form [1-3], while in other reports, it is in its multimeric form [4,5]. In general, the TNF ligands are defined by a trimeric structural homology, allowing a highly efficient receptor clustering and signal initiation mechanism [6,7]. High-affinity ligand binding induces clustering of receptors expressed in the cognate target cell that in turn initiates signal transduction pathways, culminating in cellular responses. The molecular form and expression pattern of the 'ligand' determine whether the interaction restricts or stimulates cellular activation. Depending upon the specific cellular circumstance, the outcome of this signaling may be cell proliferation, death or differentiation [6]. However, rational prediction of the cellular and physiologic outcomes initiated by an HVEM while pairing with cognate ligands remains to be elucidated. Predictive principles start with the patterns of ligand-receptor specificity and cellular expression profiles to define likely cellular interactions. According to previous studies, immune T cell-activating molecules can be divided into TNFRSF (TNF receptor SF) and IgSF (immunoglobulin SF). Interaction between the TNFR member HVEM and the IgSF member BTLA (B and T lymphocyte attenuator) represents the only pair of molecules that can communicate between the two families. Although other TNFRSFs exhibit monogamous ligand pairing with their specific TNFSF ligands, HVEM shows an extensive polygamous binding profile. Originally, HVEM was identified as the signaling ligand for CD160 by a proteomics screening of a human B cell cDNA expression library using CD160-Ig [8]. CD160 is an important signaling molecule that contributes to a wide range of immune responses, including $\mathrm{T}$ cell inhibition [8], natural killer (NK) cell activation [9] and mucosal immunity [10]. Originally, CD160 was identified as a cell surface antigen expressed on $\mathrm{NK}$ cells, CD8 ${ }^{+}$cells, a small subset of $\mathrm{CD}^{+}$cells and all intraepithelial lymphocytes [4,5,11]. Interestingly, membrane-associated LIGHT, BTLA and CD160 all activate NF- $\kappa$ B signaling downstream of HVEM following receptor engagement, whereas HVEM activates the receptors BTLA and CD160, resulting in bidirectional signaling between neighboring cells. Thus, HVEM acts as a bidirectional switch for T cell activation [12]. Other HVEM ligands, that have been identified so far, include the viral glycoprotein D (gD) encoded by herpes simplex virus (HSV) and LIGHT, which exhibits inducible expression and competes with HSV gD for HVEM. Proteinprotein interactions with HVEM or CD160 on immune cells are schematically summarized in Figure 1.

From a structural point of view, the HVEM ectodomain has two spatially distinct ligand-binding regions, one for conventional ligands (LIGHT and LT $\alpha$ ), and the second distinct site for non-canonical ligands (BTLA, CD160 and HSV gD). The cysteine-rich domain 1 (CRD1) of HVEM is essential for the binding of co-inhibitory ligands CD160 and BTLA, but not costimulatory ligand LIGHT. Deletion or blockade of HVEM CRD1 abolishes the binding of CD160 and BTLA, but not LIGHT, and converts HVEM to a dominant costimulatory molecule, possibly through the loss of negative signaling by CD160/BTLA [12]. Moreover, CD160 can also engage both classical and nonclassical MHC class I molecules, such as HLA-C, HLA-A2, HLA-B7, HLA-E and CD1d, to varying degrees [13-16]. Unlike the other MHC class I-dependent activating NK receptors, CD160 is a glycosylphosphatidylinositol (GPI)-anchored IgSF member and does not bear an immunoreceptor tyrosine-based activation (ITIM) motif. However, in human NK cells, HVEM can also promote cytolytic and pro-inflammatory pathways through CD160 as a host counter measure to human cytomegalovirus (CMV) [9]. In these studies, the viral glycoprotein UL144 (an HVEM orthologue) that mimics HVEM binding was also examined for its selectivity for BTLA. Despite the extensive sequence divergence in UL144 variants [17], BTLA bound all of them, whereas CD160 failed to bind any of them, with only one exception in which UL144 from rhesus CMV bound human and rhesus CD160 with low affinity [9]. Moreover, recent work describing CD160 deficiency in mice also confirmed its pro-inflammatory function in NK cells [18]. 
(a)

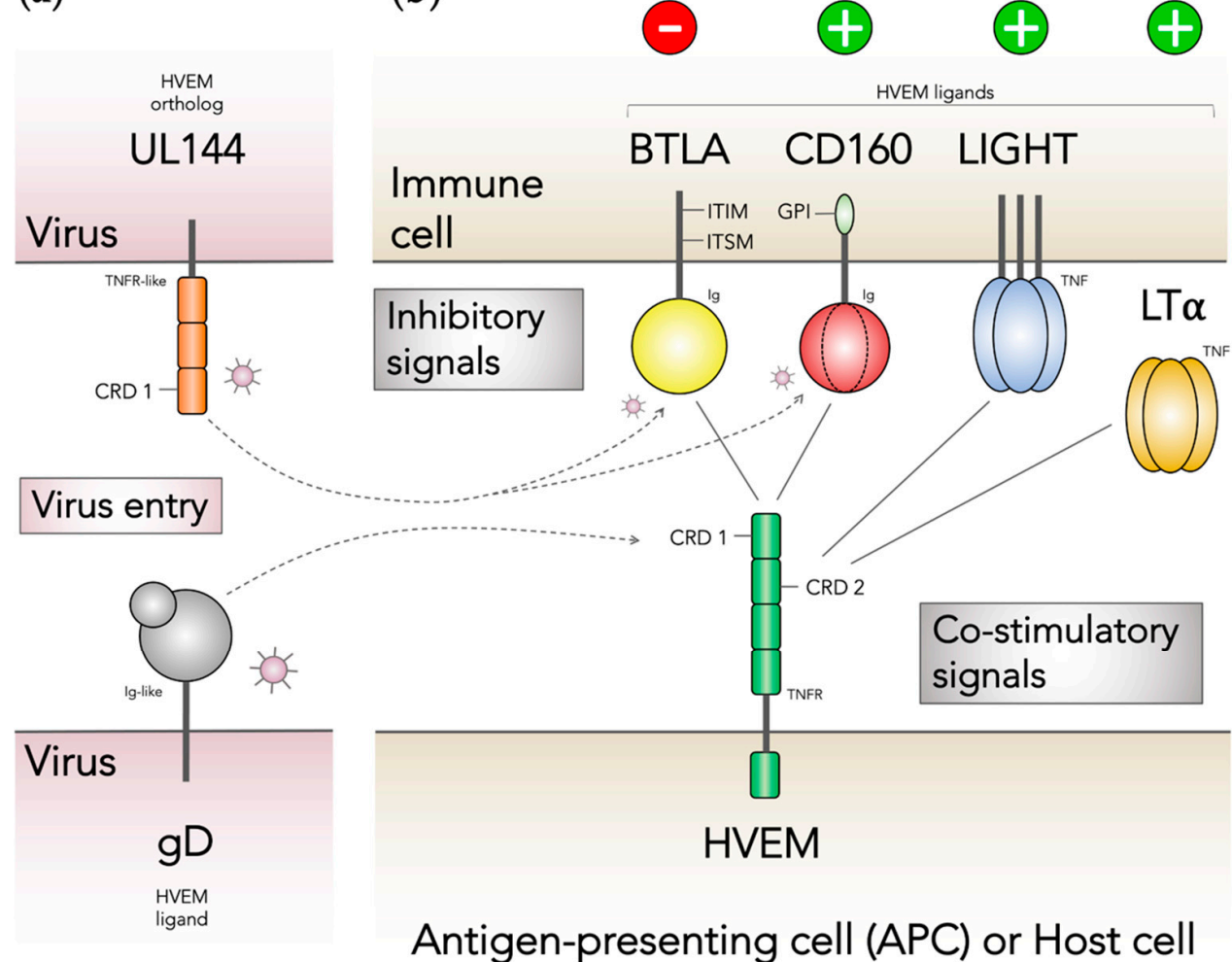

(c)
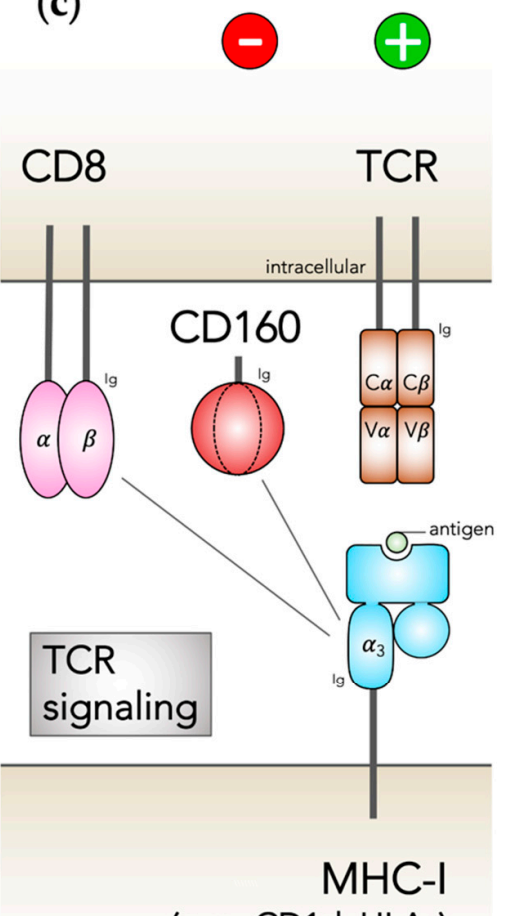

(e.g., CD1d, HLAs)

Figure 1. The HVEM- and CD160-interacting network consists of various endogenous ligands such as BTLA, LIGHT and LT $\alpha$ involved in bidirectional immune signaling (b). CD160 also influences TCR signaling by MHC class I molecules such as CD1d or various HLAs (c). The HVEM and CD160 network is influenced by two herpesviral proteins, one is glycoprotein gD (encoded by HSV), and the second one is UL144 (encoded by rhesus and human CMV) (a).

These results suggest that engagement of HVEM and activation of CD160 may serve as a critical regulatory pathway for human immune cells. Identifying the best potential targets for the development of therapeutics for inflammatory disorders is a substantial challenge because several components of this network inherently have bidirectional actions, both activating and anti-inflammatory. Accurately predicting the outcomes from blocking different molecular interactions involving HVEM and its ligands will be necessary for the development of safe and effective therapeutics to enhance immune responses and vaccination. Here, we focused on the preparation of the CD160 and HVEM protein complex as their interaction might have the most substantial potency to serve as a target for the development of various therapeutics. In particular, our study reports on the molecule preparation, characterization and initial crystallographic analysis of the CD160-HVEM complex, and both HVEM and CD160 in their ligand-free forms.

\section{Materials and Methods}

\subsection{Macromolecule Production and Characterization}

\subsubsection{Molecular Cloning}

The CD160 (324, 327 and 396 bp) and HVEM (309 bp) coding sequences were amplified by PCR from human genomic DNA and cloned into the multicloning site of the baculovirus transfer vector: pBac1-mel (Novagen) or pAc-gp67A (BD Biosciences). The coding sequences were accompanied by the $3^{\prime}$ (C-terminal) or $5^{\prime}$ (N-terminal) coding sequence of the fusion tag (OctaHis-, TwinStrep- or $\operatorname{IgG}_{1}(\mathrm{Fc})$-tag) and protease cleavage site (HRV 3C or Thrombin site) placed between the fusion tag and the gene coding sequence. For the $\operatorname{IgG}_{1}(\mathrm{Fc})$ fusion construct, the purified fragments of human $\operatorname{IgG}_{1}(680 \mathrm{bp})$ with fused N-terminal Thrombin site were subcloned into an intermediate cloning plasmid to 
place the coding sequence immediately $3^{\prime}$ to the $\mathrm{CD} 160$ and $5^{\prime}$ to the human $\operatorname{IgG}_{1}$ coding sequences. The details of the gene insert for construction of the expression plasmids are presented in Table 1 and Figure 2. Some of the expression constructs were custom made by Protein Technologies of Vienna Biocenter Core Facilities (VBCF, Vienna, Austria). All expression plasmids were propagated in E. coli JM 109 competent cells according to the manufacturer's instructions. The complete coding sequence of all plasmids was verified for absence of mutation or other modifications by sequencing prior to expression in the baculovirus expression vector system (BEVS).

Table 1. The macromolecule production information.

\begin{tabular}{|c|c|c|}
\hline Name of Protein & CD160 & HVEM \\
\hline Source Organism & \multicolumn{2}{|c|}{ Homo sapiens (Human) } \\
\hline DNA Source & \multicolumn{2}{|c|}{ Human cDNA } \\
\hline Forward Primers ${ }^{+}$ & 5'-ATTAACATCACCAGCTCAGCTTCCCAG-3' & \multirow[t]{2}{*}{ 5'-CTGCCGTCCTGCAAGGAGGACGAGTACCC-3' } \\
\hline & 3'-TGTCTCTGTGAATAGAATGGAGAAAAA-5' (27-135) & \\
\hline Reverse Primers $^{\dagger}$ & $3^{\prime}$-CCCTGTCTCTGTGAATAGAATGGAGAA-5' (27-136) & \multirow{2}{*}{ 3'-GGCGTAAGCGCGGCACGCGGCGCAGTGGTC-5' (39-142) } \\
\hline & \multirow{2}{*}{\multicolumn{2}{|c|}{$\begin{array}{r}\text { 3'-ACTGAGAGTGCCTTCATTATGGCTGA-5' (27-159) } \\
\text { pBac1-mel and/or pAc-gp67A }\end{array}$}} \\
\hline Cloning/Expression Vector & & \\
\hline Expression Host & \multicolumn{2}{|c|}{ Insect Trichoplusia ni (Hi5) and/or Spodoptera frugiperda 9 (Sf9) } \\
\hline NCBI Reference Sequence & NP_008984.1 & NP_003811.2 \\
\hline
\end{tabular}

${ }^{\dagger}$ Restriction sites are not shown. Complete information about gene construction or modifications are shown in Figure 2.

(a)

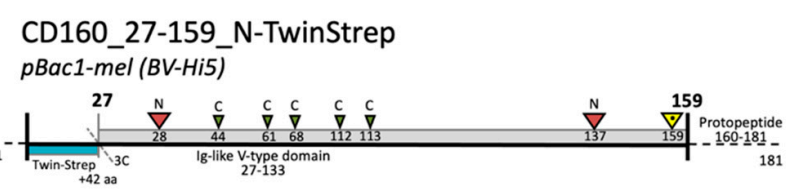

(b) CD160_27-135_C-8xHis

pAc-gp $6 \overline{7} A$ (BV-Sfg)

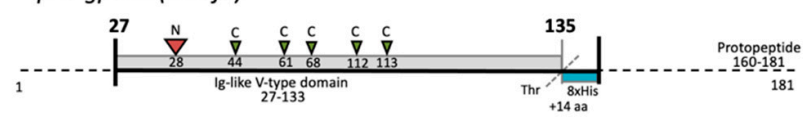

(c) CD160_27-136_C-IgG 1 (FC)

pBac1-mel and pAc-gp67A (BV-Sf9 and BV-Hi5)

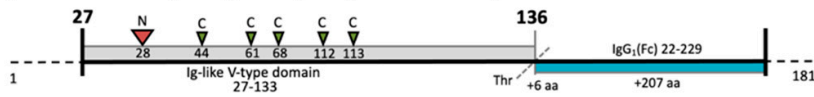

(d) HVEM_39-142_C-8xHis

pBac1-mel and pAc-gp67A (BV-Sf9 and BV-Hi5)

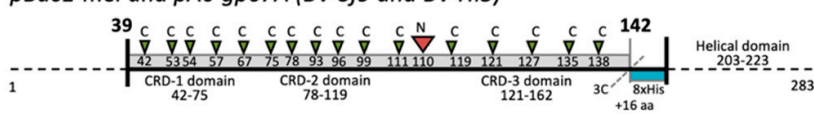

Figure 2. The details of CD160 and HVEM genes inserted in expression vectors pBac1-mel and/or pAc-gp67A. Three CD160 constructs $(\mathbf{a}-\mathbf{c})$ and one HVEM construct $(\mathbf{d})$ are reported. Cysteine $(\mathrm{C})$, asparagine $(\mathrm{N})$ and serine (S), which constitute secondary elements such as disulfide bridges, glycan attachment or lipidation, are indicated by arrows (green, red and yellow, respectively). The protease cleavage sites (dotted leaning line) and the borders of the inserted coding region (bold line), as well as domain annotations, are indicated.

\subsubsection{Protein Production and Purification}

Baculoviruses were generated by recombination-based transfection of a prepared bacmid (recombined transfer plasmid) into Hi5 and/or Sf9 cells using the ProGreen (AB Vector) or BacPAK6 (Clontech) BEVS. Cells were seeded on a T-25 flask at a concentration of $2.5 \times 10^{6}$ cells $/ \mathrm{mL}$ and cultivated in serum-free Insect-XPRESS media (Lonza) without antibiotics. To transfect cells, the culture medium was removed, and a transfection mixture containing $3 \mu \mathrm{g}$ of the baculovirus transfer vector; $0.25-0.5 \mu \mathrm{g}$ of linearized baculovirus DNA (ProGreen or BacPAK6; carrying a green fluorescent protein (GFP) reporter gene); and $20 \mu \mathrm{L}$ of transfection reagent Cellfectin (Invitrogen) or Bacfectin (Clontech) in $1 \mathrm{~mL}$ of cultivation medium was added. Following $4 \mathrm{~h}$ of cultivation, the transfection mixture was replaced with $5 \mathrm{~mL}$ of fresh Insect-XPRESS media containing $50 \mu \mathrm{g} / \mathrm{mL}$ of gentamicin (Sandoz). Transfected cells were cultivated at $27.5^{\circ} \mathrm{C}$ for 7 days. Afterwards, one or two more rounds of viral amplification (multiplicity of infection (MOI) $<1 \mathrm{PFU} /$ cell) were performed according to the manufacturer's instructions. High-titer baculovirus stocks were stored at $4{ }^{\circ} \mathrm{C}$ in the dark and subsequently used for expression assays. Each of the reported recombinant proteins was produced by infecting cells at a concentration of $2.5 \times 10^{6}$ cells $/ \mathrm{mL}$ with a freshly prepared high-titer baculovirus stock at MOI $=3-5 \mathrm{PFU} /$ cell.

For the production of the protein complex, the pool of two baculoviruses (1:1) was used to infect the cells. The cell supernatant was collected 3-5 days post-infection and clarified by centrifugation and syringe filtration over $0.45 \mu \mathrm{m}$ pores. Purification of the 
OctaHis fusion proteins was conducted by immobilized metal affinity chromatography. Supernatants were loaded on HisTrap HP prepacked columns (Cytiva) and eluted with buffer containing $50 \mathrm{mM}$ Tris $\mathrm{pH} 8.0,300 \mathrm{mM} \mathrm{NaCl}$ and imidazole at $20 \mathrm{mM}$ (wash), at a $250 \mathrm{mM}$ (elution) final concentration. Purification of the Fc fusion protein or protein complex was conducted by Protein A-based chromatography media. Supernatants were loaded on a HiTrap Protein A HP column (Cytiva) and eluted with buffer containing $20 \mathrm{mM}$ glycine $\mathrm{pH} 3$ and $50 \mathrm{mM}$ sodium chloride. The immediate addition of a high-pH buffer changed this very acidic buffer.

The Fc portion was cleaved afterwards by treating the sample with an appropriate protease according to the manufacturer's instructions. The cleaved protein or protein complex was separated from Fc on Protein A, and the collected flow through was subjected to anion-exchange chromatography using MonoQ (Cytiva) and a linear/step gradient of 0-1 M sodium chloride. Purification of the TwinStrep fusion protein was conducted by using the very high affinity of streptavidin to biotin by using liquid chromatography. In the first step of the Strep-tag purification cycle, the supernatant containing Strep-tag fusion protein was applied to a HiTrap StrepTrap HP column with immobilized StrepTactin. After the tagged protein was specifically bound, a short washing step with a physiological PBS buffer removed all other host proteins. The purified TwinStrep-tag fusion protein was gently eluted with a low concentration of desthiobiotin, which specifically competes for the biotin binding pocket. The purity of each protein was assessed by running a sample over size exclusion chromatography (SEC) with a $20 \mathrm{mM}$ HEPES and $150 \mathrm{mM} \mathrm{NaCl}$ $\mathrm{pH} 7.0$ buffer followed by Coomassie-stained reducing (using $2 \mathrm{mM}$ dithiothreitol and $2 \%$ $\beta$-mercaptoethanol) or non-reducing SDS-PAGE (Figure 3).

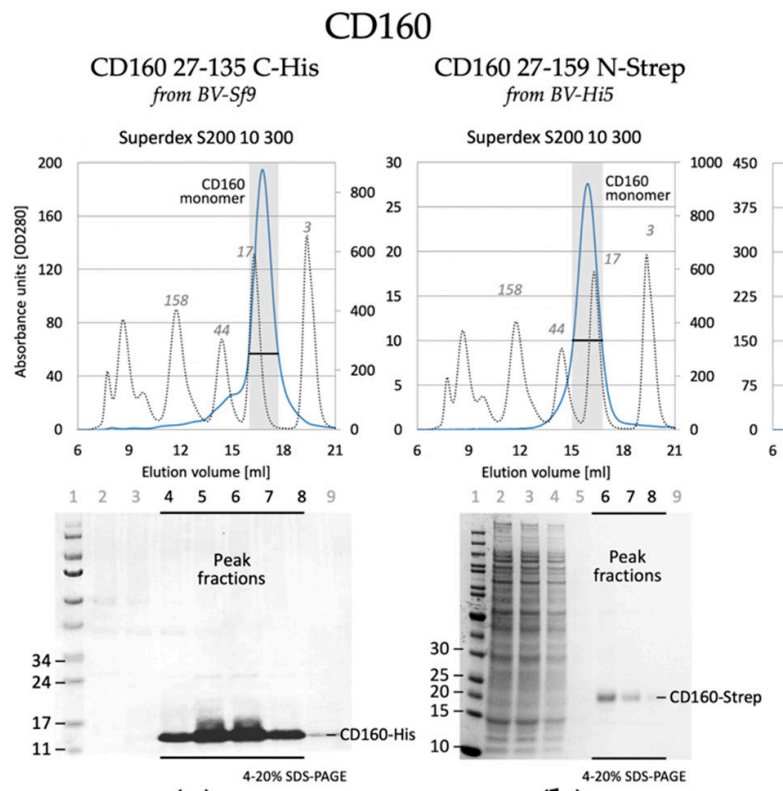

(a) (b)

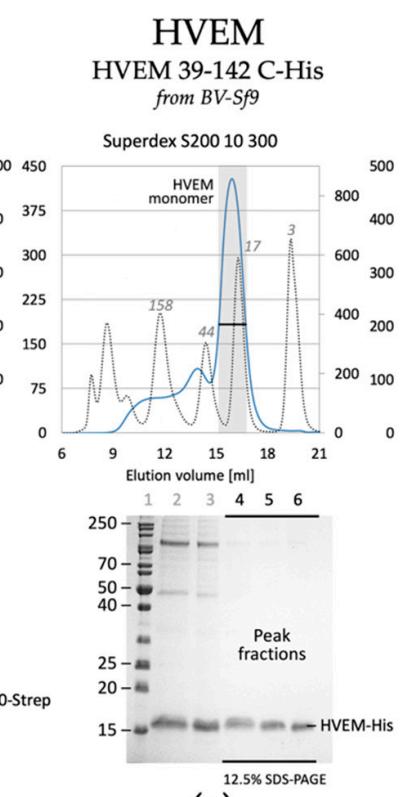

(c)



(d)

(e)

Figure 3. Purification and size estimation of studied proteins (a-d). Size exclusion chromatography (upper graphs) was performed on a Superdex S200 10/300 GL column (Cytiva), and the eluted peak fractions (bold line) were subjected to Coomassie-stained reducing SDS-PAGE (with indicated percentage of the gel). The other gel lanes, except those indicated by a bold line, contain protein samples from different purification steps (not presented here). Shaded areas in chromatograms represent CD160- and/or HVEM-containing fractions (bold line) that were pooled for crystallization and analyzed by MS. The tag-cleaved complex was subjected to anion-exchange chromatography (e) performed on a Mono Q 5/50 $1 \mathrm{~mL}$ column (Cytiva) by using a linear/step gradient of 0-1 M sodium chloride. The CD160-HVEM complex from the second peak was pooled for crystallization and checked on 4-20\% reducing SDS-PAGE (bold line).

Size exclusion (SEC) gel filtration chromatography running with a molecular protein marker in the background allowed estimation of the protein sizes indicated in chro- 
matograms. The size was calculated from the elution time of the peak fraction by using an extrapolated equation of the standard calibration curve. The purified proteins were concentrated using Vivaspin 6 (Sartorius) centrifugal concentrators with a molecular weight cutoff of $10 \mathrm{kDa}$.

\subsection{Protein Peptides Identification}

In order to characterize the recombinant proteins, we chose the well-established 'bottom-up' mass spectrometry approach, where the protein samples are digested enzymatically to produce smaller peptides that are easily characterized by mass spectrometers. This method allowed for swift identification of the expressed proteins by utilizing a combination of mass spectrometric (MS/MS) data and data analysis. The recombinant proteins produced here in insect cells were used directly or excised from SDS-PAGE (peak fractions, Figure 3) and subjected to extraction by a well-established standard method. Briefly, the small strips of gels were incubated with $0.5 \mathrm{~mL} 50 \mathrm{mM} \mathrm{AmBic}\left(\mathrm{NH}_{4} \mathrm{HCO}_{3}\right)$ buffer for $10 \mathrm{~min}$ at $21^{\circ} \mathrm{C}$ with gentle agitation. After that, the buffer was removed, and 100-200 $\mu \mathrm{L}$ of 1:1 AmBic $/ \mathrm{C}_{2} \mathrm{H}_{3} \mathrm{~N}$ was added and incubated for $30 \mathrm{~min}$. Gel strips were dehydrated in $400 \mu \mathrm{L}$ of $100 \%(v / v) \mathrm{C}_{2} \mathrm{H}_{3} \mathrm{~N}$ for $10 \mathrm{~min}$ and dried. Subsequently, the DTT solution was removed, and $100 \mu \mathrm{L}$ of $55 \mathrm{mM}$ iodoacetamide $\left(\mathrm{C}_{2} \mathrm{H}_{4} \mathrm{INO}\right.$, Sigma-Aldrich) was added (45 min in the dark). Thereafter, iodoacetamide solution was removed and gel strips were washed twice with $\mathrm{AmBic} / \mathrm{C}_{2} \mathrm{H}_{3} \mathrm{~N}$ solution, shifted to $100 \mu \mathrm{L} \mathrm{C} \mathrm{H}_{3} \mathrm{~N}$ and then rehydrated in $50 \mathrm{mM}$ AmBic buffer with $20 \mu \mathrm{g} / \mathrm{mL}$ trypsin (Promega) while set on ice for $15 \mathrm{~min}$. An additional sufficient volume of buffer was added to cover gel strips, and the reaction was incubated for $18 \mathrm{~h}$ at $37^{\circ} \mathrm{C}$. The reaction was terminated by the addition of $0.1 \%$ TFA, and the peptides were loaded onto an Acclaim PepMap 100 C18 LC column (Dionex) and separated using the HPLC Ultimate 3000 RSLC Nano modular system (Dionex). The sample was injected in a linear 30 min gradient using two mobile phases, A: $0.05 \%(v / v)$ TFA, or B: $80 \%(v / v) \mathrm{C}_{2} \mathrm{H}_{3} \mathrm{~N} ; 0.05 \%(v / v)$ TFA. The samples were analyzed by a nanoLC-MS/MS using Orbitrap Elite mass spectrometer (Thermo Fisher Scientific) in order to confirm the sequence identity of expressed proteins by peptide identification. The data were processed by the MaxQuant software package [19] with carbamidomethylation as permanent and oxidation as variable modifications.

\subsection{Crystallization}

The initial crystallization trials were carried out by the vapor diffusion technique in a sitting drop format with 24-well VDX crystallization plates (Hampton Research) using commercially available screens: PACT Premier MD 1-29 and Morpheus MD 1-46 (Molecular Dimensions). Single protein CD160 (3 mg/mL) or HVEM $(9 \mathrm{mg} / \mathrm{mL})$, or the CD160-HVEM protein complex $(3 \mathrm{mg} / \mathrm{mL})$ was mixed 1:1 with a precipitating agent with a total drop volume of $2 \mu \mathrm{L}$. All crystallization drops were equilibrated against $500 \mu \mathrm{L}$ of 1.5 M sodium chloride. Crystallization plates were incubated at a constant temperature of both $277.15 \mathrm{~K}$ and $293.15 \mathrm{~K}$, and the crystals containing CD160 or HVEM were observed at RT (293.15 K) after 10-20 or 3-5 days, respectively. Positive hits were further optimized by vapor diffusion using 24-well crystallization plates in varying protein-to-precipitant ratios (1:1, 1:2 and 2:1). The crystals were mounted separately using a nylon loop for X-ray diffraction data collection. The crystal growth parameters are summarized in Figure 4. 

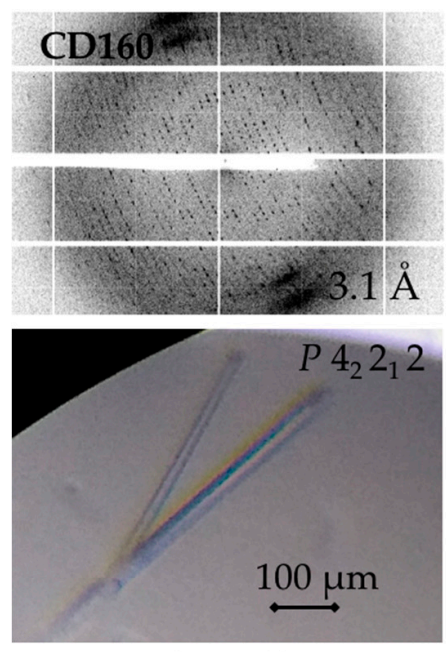

$0.2 \mathrm{M} \mathrm{Na}$ malonate dibasic monohydrate $\mathrm{pH} 6.5$ $0.1 \mathrm{M}$ Bis-Tris propane 20\% (w/v) PEG 3350

(a)

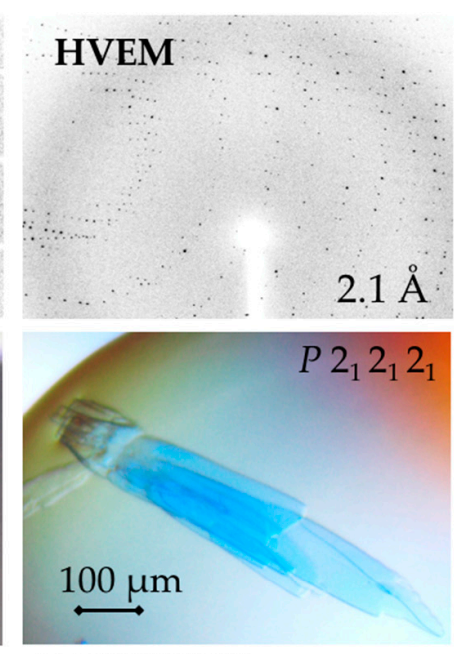

$0.15 \mathrm{M}$ HEPES 7.5

$0.15 \mathrm{M}$ sodium chloride $0.05 \mathrm{M}$ ammonium sulfate $30 \%(\mathrm{w} / \mathrm{v})$ PEG 8000 $5 \%$ (v/v) Glycerol
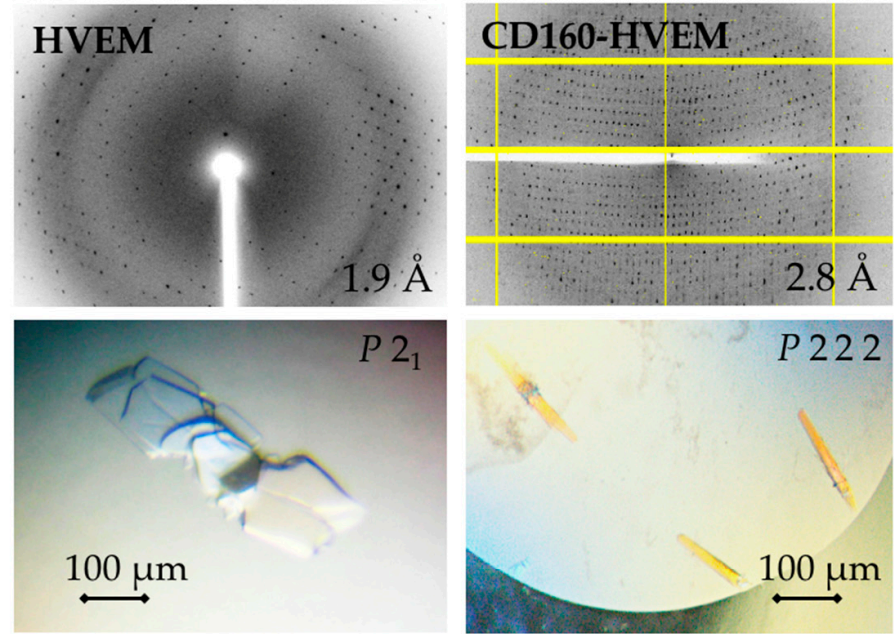

$0.15 \mathrm{M}$ calcium chloride 15\% (w/v) PEG 3300 $30 \%$ (w/v) PEG 8000 $5 \%$ (v/v) Glycerol

(c)

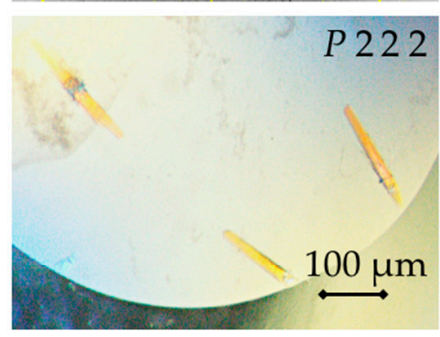

$0.2 \mathrm{M}$ ammonium chloride 0.1 M HEPES $\mathrm{pH} 8.0$ 20\% (w/v) PEG 6000 (b)

Figure 4. Diffraction images of CD160 (a), HVEM (b,c) and CD160-HVEM (d) crystals with indicated resolution (upper row of images). The particular crystals tested for diffraction (lower row of images) with indicated space group, crystal size and crystallization conditions. The DNA expression constructs used for the protein production and subsequent crystallization are as follows: CD160 (a) was produced by the construct detailed in Figure 2c (with cleaved tag); both HVEM crystals $(\mathbf{b}, \mathbf{c})$ were produced by one DNA construct detailed in Figure 2d; and the CD160-HVEM complex (d) was produced by co-expression of two constructs detailed in Figure 2c (with cleaved tag) and Figure 2d.

\subsection{Data Collection and Processing}

Mounted cryo-loops (Hampton Research) were used to separate and fish out the crystals from the drop. Several cryoprotectants were tested for the best cryogenic conditions, including glycerol, ethylene glycol, polyethylene glycol 400 and 2-methyl-2,4-pentane-diol. The best condition was achieved by cryoprotection in well solutions containing $25 \%(v / v)$ glycerol, except those containing cryoprotective formulations in precipitating conditions, and then flash cooled in liquid nitrogen for data collection at $100 \mathrm{~K}$. The quality of diffraction was tested and the complete datasets were collected on Diamond Light Source beamline I04 (UK) or SSRL beamline BL7-1 (USA). The parameters for individual data collection for crystals of CD160, HVEM (two crystal forms) and CD160-HVEM are shown in Table 2. The data were processed using XDS [20] and merged and scaled using AIMLESS in CCP4 [21].

Table 2. Data collection statistics and cell parameters for crystals of CD160 and HVEM (two crystal forms) and for the protein complex CD160-HVEM.

\begin{tabular}{|c|c|c|c|c|}
\hline & CD160 & \multicolumn{2}{|c|}{ HVEM } & CD160-HVEM \\
\hline \multirow{3}{*}{ Diffraction source } & Synchrotron & \multicolumn{2}{|c|}{ Synchrotron } & Synchrotron \\
\hline & DIAMOND & \multicolumn{2}{|c|}{ SSRL } & DIAMOND \\
\hline & BL-I04 & \multicolumn{2}{|c|}{ BL7-1 } & BL-I04 \\
\hline Wavelength $(\AA)$ & 1.281 & 0.978 & 0.975 & 1.282 \\
\hline Temperature (K) & \multicolumn{4}{|c|}{100} \\
\hline \multirow{2}{*}{ Detector } & PIXEL Dectris & CCD ADSC & CCD ADSC & PIXEL Dectris \\
\hline & Eiger2 X 16M & Quantum 315 & Quantum 315 & Eiger X 16M \\
\hline $\begin{array}{l}\text { Crystal-to-detector } \\
\text { distance }(\mathrm{mm})\end{array}$ & 207 & 200 & 200 & 222 \\
\hline $\begin{array}{l}\text { Rotation range } \\
\text { per image }\left(^{\circ}\right)\end{array}$ & 0.1 & 1 & 1 & 0.15 \\
\hline Total rotation range $\left(^{\circ}\right)$ & \multicolumn{4}{|c|}{360} \\
\hline
\end{tabular}


Table 2. Cont.

\begin{tabular}{|c|c|c|c|c|}
\hline \multirow[b]{2}{*}{$\begin{array}{l}\text { Exposure time } \\
\text { per image (s) }\end{array}$} & \multirow{2}{*}{$\begin{array}{c}\text { CD160 } \\
0.050\end{array}$} & \multicolumn{2}{|c|}{ HVEM } & \multirow{2}{*}{$\begin{array}{c}\text { CD160-HVEM } \\
0.010\end{array}$} \\
\hline & & 0.100 & 0.100 & \\
\hline Space group & $P 4_{2} 2_{1} 2$ & $P 2_{1} 2_{1} 2_{1}$ & $P 2_{1}$ & $P 222$ \\
\hline$a, b, c(\AA)$ & $122.22,122.22,154.79$ & $47.21,62.42,103.57$ & $50.19,47.51,67.29$ & $74.05,94.14,140.52$ \\
\hline$\alpha, \beta, \gamma\left({ }^{\circ}\right)$ & $90,90,90$ & $90,90,90$ & $90,106.69,90$ & $90,90,90$ \\
\hline Mosaicity $\left(^{\circ}\right)$ & - & - & - & 0.14 \\
\hline Resolution range $(\AA)$ & $\begin{array}{l}3.12-75.46 \\
(3.12-3.17)\end{array}$ & $\begin{array}{l}2.10-20.0 \\
(2.10-2.16)\end{array}$ & $\begin{array}{l}1.90-20.00 \\
(1.90-1.98)\end{array}$ & $\begin{array}{l}2.84-29.10 \\
(2.84-2.91)\end{array}$ \\
\hline Redundancy & 26.4 & 7.6 & 8.6 & 19.8 \\
\hline Unique reflections & 21562 & 17307 & 23026 & 23783 \\
\hline Completeness (\%) & $100.0(100.0)$ & $98.8(97.4)$ & $99.25(96.22)$ & $99.10(88.80)$ \\
\hline $\mathrm{CC}$ half $^{+}$ & $1.0(0.39)$ & $1.0(0.63)$ & $1.0(0.66)$ & $1.0(0.80)$ \\
\hline Mean $I / \sigma(I)$ & $9.1(0.3)$ & $8.1(2.7)$ & $12.8(2.2)$ & $20.9(2.7)$ \\
\hline$R$ factor r.i.m. $(\%) \ddagger$ & $0.334(6.932)$ & $0.132(0.976)$ & $0.182(0.875)$ & $0.134(0.981)$ \\
\hline $\begin{array}{l}\text { Overall } B \text { factor from } \\
\text { Wilson plot }\left(\AA^{2}\right)\end{array}$ & 87.65 & 21.66 & 19.13 & 16.04 \\
\hline
\end{tabular}

${ }^{\dagger} \mathrm{CC}$ half is correlation coefficient of half datasets. $\ddagger$ The redundancy-independent merging $\mathrm{R}$ factor is reported. () Parentheses indicate statistics for the highest resolution shell.

\section{Results and Discussion}

\subsection{Purification and Characterization of CD160, HVEM and Their Complex}

Our initial efforts to prepare CD160 and HVEM in a properly folded form have failed many times in the inexpensive and well-known prokaryotic expression system E. coli, probably due to the highly reduced environment. Nevertheless, some reports are currently available on the production of these proteins in prokaryotic hosts [1,2]. Our more intensive expression studies showed that CD160 produced by constructs (a), (b) and (c) detailed in Figure 2 and HVEM (d) are efficiently expressed in BEVS with various solubility and affinity tags (N-TwinStrep, C-OctaHis and $\mathrm{C}-\mathrm{IgG}_{1}$, respectively) in sufficient quantity and at a high quality for mass spectrometry and crystallographic studies. Moreover, the BEVS allowed for eukaryotic posttranslational modifications without complication. To confirm the molecular appearance of the heterologously expressed proteins, CD160, HVEM and their complex were purified by affinity and gel filtration chromatography (Figure 3a-d). The CD160-HVEM protein complex was, after cleavage of the fusion tag, purified with an additional step of anion-exchange chromatography to obtain the desired quality, thus distinguishing the unwanted complex formations of different net surface charged samples (Figure 3e). The final SEC indicates that CD160 ectodomains I27-T135 and I27-S159 produced by constructs (a) and (b) (Figure 2) exist as a single monodisperse species with a molecular size of about $15 \mathrm{kDa}$ and $22 \mathrm{kDa}$, respectively (Figure 3a,b). Fractions across the entire peak were subjected to SDS-PAGE analysis under reducing and non-reducing conditions. All fractions migrated with an approximate molecular weight predicted for the monomeric CD160 including tags, suggesting that CD160 expressed with these short fusion tags (OctaHis or TwinStrep) exists as a monomer in solution. However, we further investigated the effect of various tags and fusion partners on the overall fitness of the sample. To confirm that the expressed CD160-HVEM protein complex reflects the behavior of the native material, we co-expressed the human CD160 I27-G136 produced by construct (c) together with the human HVEM L39-A142 (d) in BEVS (Figure 2). The final purified CD160-HVEM complex (tag cleaved) was subjected to SEC analysis at a concentration of approximately $6 \mathrm{mg} / \mathrm{mL}$. The complex appeared as two separated peaks at molecular sizes of about $78 \mathrm{kDa}$ and $19 \mathrm{kDa}$, estimated by SEC, and approximately $\sim 80 \mathrm{kDa}$ and $\sim 17 \mathrm{kDa}$, estimated by the corresponding SDS-PAGE analysis under reducing and nonreducing conditions (Figure 3d). The estimated molecular sizes correspond to the calculated molecular weights of trimeric CD160 and monomeric HVEM, respectively. These studies demonstrate that CD160-HVEM undergoes molecular separation on a gel filtration column 
apparently due to the low binding affinity between CD160 and HVEM molecules. This was also observed by others [1], also showing SEC traces of the CD160-HVEM complex in different molar ratios. These results together indicate that CD160 and HVEM do not form a stable complex in solution under these conditions: SEC running in $20 \mathrm{mM}$ HEPES, $150 \mathrm{mM}$ $\mathrm{NaCl}, \mathrm{pH}$ 7.0. However, we further examined the complex formation under different conditions by subjecting the CD160-HVEM complex to anion-exchange chromatography. High-resolution separation by the net surface charge of the sample was demonstrated at $\mathrm{pH} 8$ due to an anion gradient of $1 \mathrm{M}$ sodium chloride that yielded several peaks all containing the complex. The elution peak of CD160-HVEM at a concentration of about $15 \%$ was collected and subjected to SDS-PAGE and MS analysis. The protein migration on SDS-PAGE was similar under reducing and non-reducing conditions and corresponded to the mass of the CD160 trimer ( $\sim 80 \mathrm{kDa})$ and HVEM monomer $(\sim 17 \mathrm{kDa})$. CD160 could not be reduced by common reducing agents (DTT or $\beta-\mathrm{ME}$ ), suggesting that the subunits of the CD160 multimer are probably held together through strong inter- or intramolecular forces of the flexible strands. These findings are consistent with the previously reported behavior of the full-length CD160 [5], migrating with an approximate molecular mass of $80 \mathrm{kDa}$ (under both non-reducing and reducing conditions), that was endogenously expressed on primary cells and immunoprecipitated with specific CD160 monoclonal antibodies. Together, these results support the existence of the physiological multimeric form of the CD160 ectodomain co-expressed here in a complex with HVEM.

The final samples of all proteins and complexes reported here were over $99 \%$ pure, as estimated by the corresponding SDS-PAGEs. The final yields of CD160-C-His and -N-Strep were approximately 1 and $0.5 \mathrm{mg} / \mathrm{L}$ of culture media, respectively. These proteins were concentrated to a final concentration of $3 \mathrm{mg} / \mathrm{mL}$. The final yields of $\mathrm{CD} 160-\mathrm{C}-\mathrm{IgG} \mathrm{G}_{1}(\mathrm{Fc})$, HVEM-C-His and their protein complex were approximately $3-5 \mathrm{mg} / \mathrm{L}$ of culture media. The proteins were concentrated to the final concentrations of 3,9 and $3 \mathrm{mg} / \mathrm{mL}$, respectively, and used for mass spectrometry and crystallographic studies. Macromolecule production information is summarized in Table 1 and Figures 2 and 3.

\subsection{Peptide Identification of CD160 and HVEM by Mass Spectrometry}

As many proteins expressed in recombinant hosts often show different properties than those expected, we sought to determine whether the appearance of CD160 in multimeric form reflects the expected gene sequence and behavior of the native material, as well as whether the HVEM construct is produced in the expected form. Therefore, we performed several checkpoint methods and quality controls to prove the correctness of the expressed recombinant proteins prior to crystallographic studies. To obtain proper assurance of the protein identity, all variants of CD160, HVEM and their complex produced here were analyzed by mass spectrometry. As peptide mapping is the principal technique for confirming a protein's primary structure, or at least part of the amino acid sequence, we first subjected the purified proteins and/or gel bands to tryptic digestion. For all the expressed proteins, we confirmed up to five-seven peptides (with 100\% identity) that correspond to a sequence coverage of 30-43\% for the CD160 monomer produced by constructs (a) and (b) and about $45 \%$ for HVEM (d) (Figure 2), all including the C- or N-terminal anchor sequences (TwinStrep or OctaHis). About 47\% sequence coverage for the CD160 trimer produced by construct (c) (Figure 2) was confirmed by the presence of six peptides (representative peptides are shown in (c), Figure S1). Furthermore, from the extracted gel band of the final fraction of the CD160 trimer (Figure 3e, SDS-PAGE upper band) that was also subjected to analysis of peptide fragments derived from trypsin digestion, it was revealed that the CD160 extracellular region starts with the sequence ${ }^{27}$ INITS $^{31}$, which is consistent with the previous findings of $[1,2,22]$. This analysis also confirmed that the extracellular domain of CD160 contains five cysteines (C44, C61, C68, C112 and C113) after cleavage of the signal sequence and two confirmed N-linked glycosylation sites (N28 and N137). 
3.3. Crystallization of Native (Non-Linked) CD160-HVEM Complex and Both Proteins in the Absence of Their Binding Partner

Many efforts from several research groups that aimed to crystallize the CD160-HVEM complex have failed, perhaps due to the low affinity between these molecules (data not shown). To circumvent the challenges associated with low affinity, recently, Liu et al. 2019 [1] designed a covalent single-chain CD160-HVEM construct in which the C-terminus of CD160 was covalently fused to the N-terminus of HVEM via a (GGGGS) ${ }^{4}$ linker. The single-chain CD160-HVEM protein was produced using Drosophila S2 cells, purified to homogeneity and crystallized in space group $I_{1} 22$ as a monomer. However, considering the fact that CD160 might co-exist as a multimer in solution, we sought to produce and crystallize the native (non-linked) CD160-HVEM protein complex to determine its molecular composition. The protein complex was produced in BEVS and purified to homogeneity. Three-dimensional crystals of the CD160-HVEM protein complex were grown using a precipitant solution containing: $0.2 \mathrm{M}$ ammonium chloride, $0.1 \mathrm{M}$ HEPES pH 8.0, 20\% $(w / v)$ PEG 6000, and 0.2 M ammonium chloride, $0.1 \mathrm{M}$ sodium acetate $\mathrm{pH} 5.0,20 \%(w / v)$ PEG 6000. Improved crystals were subsequently obtained in the first conditions mentioned above, but with a 2:1 protein-to-precipitant ratio. The final condition that yielded CD160-HVEM crystals (Figure 4d) suitable for X-ray diffraction measurement was $0.2 \mathrm{M}$ ammonium chloride, 0.1 M HEPES pH 8.0, 20\% (w/v) PEG 6000. The purified CD160 and HVEM proteins alone were also tested in various crystallization conditions, although CD160 alone (produced by DNA constructs $(a-c)$ detailed in Figure 2) hardly produced crystals suitable for X-ray diffraction, while purified HVEM (free of ligand) produced many crystals in various conditions. A solution consisting of $200 \mathrm{mM}$ sodium malonate dibasic monohydrate $\mathrm{pH}$ 6.5, $100 \mathrm{mM}$ Bis-Tris propane and 20\% (w/v) PEG 3350 was eventually found and optimized to produce CD160 (construct in Figure 2c, with cleaved tag) crystals of an elongated shape with rather borderline quality diffraction (Figure 4a). Purified HVEM crystalizes in various conditions (Figure 4b,c), accommodating different outer shapes (mainly as multiple crystals), as well as space groups, but is diffracted to a far higher resolution. The crystals of CD160, HVEM and their complex employed in the $X$-ray diffraction experiments grew to maximum dimensions of approximately $0.8 \times 0.05$ $\times 0.05 \mathrm{~mm}, 0.9 \times 0.15 \times 0.05 \mathrm{~mm}$ and $0.1 \times 0.03 \times 0.03 \mathrm{~mm}$, respectively (Figure 4 ).

\subsection{Initial X-ray Analysis of the CD160, HVEM and CD160-HVEM Crystals}

Some of the presented crystallization conditions already contain the necessary cryoprotection. For all others, the optimized cryoprotection strategy involved soaking the crystals sequentially for 30-60 s in a reservoir solution supplemented with $25 \%(v / v)$ glycerol. Although some diffraction patterns with smeared spots were observed in the best CD160 crystals, the diffraction data collection turned out to be feasible, and the crystals diffracted to a resolution of $3.1 \AA$. The crystals belonged to the tetragonal space group $P 4{ }_{2} 2{ }_{1} 2$ with unit cell parameters $a=122.22 \AA, b=122.22 \AA$ and $c=154.79 \AA$. A complete $\mathrm{X}$-ray diffraction dataset was collected with a minimum Bragg spacing of $3.12 \AA$. A representative frame recorded during this data collection is shown in Figure 4a. The volume of the asymmetric unit allows the presence of a multimer, producing a Matthews [23] volume $\left(V_{M}\right)$ of $3.5 \AA^{3} \mathrm{Da}^{-1}$ and a solvent content of $65.1 \%$. The diffraction data for two HVEM crystal forms were collected at $1.90 \AA$ and $2.10 \AA$ resolutions that, at present, refer to the highest reported resolutions in the PDB database. The crystals belonged to orthorhombic $P 2{ }_{1} 2{ }_{1} 2_{1}$ and monoclinic $P 2{ }_{1}$ space groups with the unit cell parameters $a=47.21 \AA$, $b=62.42 \AA$ and $c=103.57 \AA$, and $a=50.19 \AA, b=47.51 \AA, c=67.29 \AA$ and $\beta=106.69^{\circ}$, respectively. For both crystal forms, the presence of two molecules in the asymmetric unit was assumed by calculating the $V_{M}$ as $2.12 \AA^{3} \mathrm{Da}^{-1}$ and $2.13 \AA^{3} \mathrm{Da}^{-1}$, respectively. The corresponding solvent content was $41.97 \%$ and $42.38 \%$. The local symmetry connecting the protein molecules in the asymmetric unit was investigated using MOLREP [24] from the CCP4 package [25]. It is also compatible with the presence of two monomers in an asymmetric unit of the crystal. Phasing based on diffraction data from both crystal forms 
revealed two independent molecules of HVEM in the asymmetric unit, while superposition of the two chains showed similar structures in both cases, with a root mean square deviation (RMSD) of less than $0.5 \AA$ for 98 aligned $\mathrm{C} \alpha$ atoms. The highly purified CD160-HVEM complex separated from the differently charged sample by an anion gradient, and the yielded elongated-shape crystals exhibited diffraction consistent with the orthorhombic space group P222, which extended to a resolution of $2.84 \AA$. The unit cell parameters could accommodate one trimeric complex (3:3) assumed by calculating the $\mathrm{V}_{\mathrm{M}}$ as $1.99 \AA^{3}$ $\mathrm{Da}^{-1}$, with a corresponding solvent content of $38.22 \%$. Phasing based on CD160-HVEM diffraction data is now being carried out with CD160 and HVEM serving as the search models with PDB codes $6 \mathrm{NG} 9$ and $4 \mathrm{FHQ}$, respectively [1,22]. The data collection statistics and unit cell parameters are reported in Table 2.

In conclusion, the native non-linked CD160-HVEM complex here was co-expressed in BEVS and finally purified to homogeneity by anion-exchange chromatography to provide missing evidence of the trimeric form in solution. Its trimeric existence was also confirmed by initial crystallographic analysis. The native CD160-HVEM complex crystallized in a space group with unit cell parameters evidently providing ample space for the multimeric form.

\subsection{Functional Implications of Trimeric CD160 Occurring in Solution}

CD160 was discovered in a program in the early 1990s to identify natural killer (NK) cell-specific receptors by immunization of mice with a human NK leukemia (cell line YT2C2), which resulted in the NK-specific IgM monoclonal antibody (mAb) BY55 [5]. The BY55 mAb was used to clone the complementary DNA (cDNA) encoding the gene, and this was later assigned the CD160 designation [4]. The mRNA encodes an Ig domain membrane protein with two different anchors to the cell surface, a glycosphingolipid (GPI) anchor and a transmembrane form [26], generated by alternate splicing. CD160 may also be found as a soluble molecule as a result of cleavage of the GPI-linked form by phospholipases. Two $\mathrm{mAbs}$ (CL1-R2 and BY55) recognizing the CD160 isoform (including the GPI anchor) did not react with the other CD160 isoform (including the transmembrane domain), suggesting a different structural composition of CD160 being engaged. These data collectively conclude the oligomeric existence of CD160, allowing for various antibody specificities. Moreover, the CD160 GPI cDNA sequence predicts a glycosylated protein of about 20-25 kDa, whereas the native CD160 antigen on NK cells is $80 \mathrm{kDa}[4,5]$. This difference in the expected size was assumed to be a result of the reduction-resistant multimerization of the CD160 protein by the four additional cysteines besides the two IgSF consensus cysteines [12]. However, later crystallographic studies in 2019 disapproved the involvement of some cysteines in intramolecular formation [1]. These authors showed that two disulfide bonds are present in the CD160 structure, including the consensus IgSF disulfide formed between C44 and C112 and a non-canonical disulfide bond between C61 and C68. An unpaired cysteine, C113, was observed to be inaccessible to the solvent, suggesting that it is probably not essential for the integrity of the CD160 structure. However, some immunoglobulin domains can undergo strand swapping, which could possibly alter the structure to expose the unpaired cysteine in some cases, and this could be the case of the CD160 trimer. Evidence from a previous report [1,2] indicates that the CD160 ectodomain is present in monomeric form; however, early evidence using monoclonal antibody BY55 [4,5], as well as our new biochemical and preliminary X-ray data, suggested that CD160 forms a multimer beside the monomeric form. In addition, CD160 was already known to be a marker of activated endothelial cells that surround newly formed blood vessels and, as such, is considered a potential target for the treatment of diseases associated with neoangiogenesis [27]. It was found that anti-CD160 is a potent inhibitor of ocular vascularization [28]; thus, ongoing studies may reside in searching for a new potent antibody against oligomeric CD160.

The monomeric structure of CD160 reported by [1] resembles the structure of BTLA, while covalently linked single-chain CD160-HVEM is in a 1:1 stoichiometry. This is in contrast to the 3:3 stoichiometry of the LIGHT-to-HVEM assembly, suggesting the covalently 
linked single-chain CD160-HVEM could accommodate a forced 1:1 stoichiometry instead. The CD160 interaction with HVEM as well as with MHC-I molecules has been described by previous functional studies. For example, both CD160 and BTLA can bind to HVEM in cis form to inhibit T cell activation, whereas LIGHT does not bind to HVEM in the cis form [29]. The engagement of CD160 receptors at the cell surface leads to the production of cytokines and the induction of cytotoxic functions $[14,15]$. In addition, the CD160 receptor appears to be unique, as it is encoded by a gene located on human chromosome 1 and corresponds to a GPI-anchored Ig-like molecule in which surface expression is rapidly down-modulated upon NK cell activation [30]. Given that various HLA class I molecules may also bind to CD160 [13,14], it cannot be excluded that the other important soluble molecules, such as MHC class I, could also trigger this receptor to exert antiangiogenic functions. Additionally, due to the fact that the CD160 trimer forms only under certain conditions or on a specific cell type, $\mathrm{pH}$-dependent formation also cannot be excluded in this case. Moreover, in humans, both ligands and receptors often show idiosyncratic posttranslational processing mechanisms, e.g., cleavage of membrane ligands into soluble proteins, which dramatically alters the communication loop from a closed cell-to-cell contact mechanism to a potential systemic impact [6]. Therefore, there has been considerable effort made toward mapping the CD160-mediated interactions occurring on immune cells by producing relevant molecules either in a soluble recombinant form (with solubility tags; e.g., OctaHis, $\operatorname{IgG}_{1}\left(\mathrm{Fc}_{\mathrm{C}}\right)$, TwinStrep, FLAG; or labeled otherwise) or as cell-anchored versions (e.g., 293T-expressing). The diversity of intracellular signaling cascades set in motion by receptor activation creates dynamic diversity, further lessening the accuracy of any predictive attempts. Predictive capability remains a goal that has important consequences for interpreting results in physiologic models and human clinical trials. Thus, the HVEM and CD160 interacting network consisting of endogenous molecules BTLA, LIGHT and LT $\alpha$, and also viral glycoproteins, e.g., gD (encoded by HSV) and UL144 (encoded by CMV) (Figure 1), allows for the interpretation of the complex outcomes of these multicellular interactions.

Supplementary Materials: The following are available online at https:/ / www.mdpi.com/article/10 .3390 / cryst11070820/s1, Figure S1: CD160 peptide identification by mass spectrometry.

Author Contributions: Conceptualization: I.N.; protein production: S.L., M.B., R.Š. and I.N.; mass spectrometry: M.N., S.L. and I.N.; crystallization and structural experiments: I.N. and S.L.; data collection: I.N. and S.L.; analysis of the results: I.N. and S.L.; writing manuscript: I.N. and S.L.; review and editing manuscript: I.N., S.L. and D.M.Z.; resources: I.N. and D.M.Z.; supervision (incl. external): I.N. and D.M.Z.; funding acquisition: I.N. All authors have read and agreed to the published version of the manuscript.

Funding: This research was funded by the contribution of the Slovak Research and Development Agency under the project APVV-14-0839 and continuous project APVV-19-0376; and the contribution of the Scientific Grant Agency of the Slovak Republic under the grant VEGA-02/0020/18 and VEGA02/0060/21. IN was Marie Curie Fellow financed by program SASPRO co-funded by the European Union and the Slovak Academy of Sciences. Part of the research team was supported by the Interreg V-A SK-AT cooperation program by project CAPSID under the contract No. NFP305010V235 cofinanced by the European Regional Development Fund. Part of this work was supported by a grant from the Ministry of Health of the Slovak Republic under the project registration number 2019/7-CHÚSAV-4.

Institutional Review Board Statement: Not applicable.

Informed Consent Statement: Not applicable.

Data Availability Statement: Not applicable.

Acknowledgments: The authors would like to thank Diamond Light Source for beamtime and the staff of beamlines I04 for assistance with crystal testing and data collection. Use of the Stanford Synchrotron Radiation Lightsource, SLAC National Accelerator Laboratory, is supported by the U.S. Department of Energy, Office of Science, Office of Basic Energy Sciences under Contract No. DE- 
AC02-76SF00515. The SSRL Structural Molecular Biology Program is supported by the DOE Office of Biological and Environmental Research, and by the National Institutes of Health and National Institute of General Medical Sciences (including P30GM133894). The contents of this publication are solely the responsibility of the authors and do not necessarily represent the official views of NIGMS or NIH. The some of the expression constructs were custom made by the Protein Technologies Facility at Vienna BioCenter Core Facilities (VBCF), member of the Vienna BioCenter (VBC), Austria.

Conflicts of Interest: The authors declare no conflict of interest.

\section{References}

1. Liu, W.; Garrett, S.C.; Fedorov, E.V.; Ramagopal, U.A.; Garforth, S.J.; Bonanno, J.B.; Almo, S.C. Structural basis of CD160: HVEM recognition. Structure 2019, 27, 1286-1295 e4. [CrossRef]

2. Kojima, R.; Kajikawa, M.; Shiroishi, M.; Kuroki, K.; Maenaka, K. Molecular basis for herpesvirus entry mediator recognition by the human immune inhibitory receptor CD160 and its relationship to the cosignaling molecules BTLA and LIGHT. J. Mol. Biol. 2011, 413, 762-772. [CrossRef] [PubMed]

3. Stiles, K.M.; Whitbeck, J.C.; Lou, H.; Cohen, G.H.; Eisenberg, R.J.; Krummenacher, C. Herpes simplex virus glycoprotein D interferes with binding of herpesvirus entry mediator to its ligands through downregulation and direct competition. J. Virol. 2010, 84, 11646-11660. [CrossRef] [PubMed]

4. Anumanthan, A.; Bensussan, A.; Boumsell, L.; Christ, A.D.; Blumberg, R.S.; Voss, S.D.; Patel, A.T.; Robertson, M.J.; Nadler, L.M.; Freeman, G.J. Cloning of BY55, a novel Ig superfamily member expressed on NK cells, CTL, and intestinal intraepithelial lymphocytes. J. Immunol. 1998, 161, 2780-2790. [PubMed]

5. Maiza, H.; Leca, G.; Mansur, I.G.; Schiavon, V.; Boumsell, L.; Bensussan, A. A novel 80-kD cell surface structure identifies human circulating lymphocytes with natural killer activity. J. Exp. Med. 1993, 178, 1121-1126. [CrossRef]

6. Ward-Kavanagh, L.K.; Lin, W.W.; Sedy, J.R.; Ware, C.F. The TNF receptor superfamily in co-stimulating and co-inhibitory responses. Immunity 2016, 44, 1005-1019. [CrossRef]

7. Bodmer, J.L.; Schneider, P.; Tschopp, J. The molecular architecture of the TNF superfamily. Trends Biochem. Sci. 2002, 27, 19-26. [CrossRef]

8. Cai, G.; Anumanthan, A.; Brown, J.A.; Greenfield, E.A.; Zhu, B.; Freeman, G.J. CD160 inhibits activation of human CD4+ T cells through interaction with herpesvirus entry mediator. Nat. Immunol. 2008, 9, 176-185. [CrossRef]

9. Sedy, J.R.; Bjordahl, R.L.; Bekiaris, V.; Macauley, M.G.; Ware, B.C.; Norris, P.S.; Lurain, N.S.; Benedict, C.A.; Ware, C.F. CD160 activation by herpesvirus entry mediator augments inflammatory cytokine production and cytolytic function by NK cells. J. Immunol. 2013, 191, 828-836. [CrossRef]

10. Shui, J.W.; Larange, A.; Kim, G.; Vela, J.L.; Zahner, S.; Cheroutre, H.; Kronenberg, M. HVEM signalling at mucosal barriers provides host defence against pathogenic bacteria. Nature 2012, 488, 222-225. [CrossRef] [PubMed]

11. Nikolova, M.; Marie-Cardine, A.; Boumsell, L.; Bensussan, A. BY55/CD160 acts as a co-receptor in TCR signal transduction of a human circulating cytotoxic effector T lymphocyte subset lacking CD28 expression. Int. Immunol. 2002, 14, 445-451. [CrossRef] [PubMed]

12. Cai, G.; Freeman, G.J. The CD160, BTLA, LIGHT/HVEM pathway: A bidirectional switch regulating T-cell activation. Immunol Rev. 2009, 229, 244-258. [CrossRef] [PubMed]

13. Agrawal, S.; Marquet, J.; Freeman, G.J.; Tawab, A.; Bouteiller, P.L.; Roth, P.; Bolton, W.; Ogg, G.; Boumsell, L.; Bensussan, A. Cutting edge: MHC class I triggering by a novel cell surface ligand costimulates proliferation of activated human $\mathrm{T}$ cells. J. Immunol. 1999, 162, 1223-1226. [PubMed]

14. Barakonyi, A.; Rabot, M.; Marie-Cardine, A.; Aguerre-Girr, M.; Polgar, B.; Schiavon, V.; Bensussan, A.; Le Bouteiller, P. Cutting edge: Engagement of CD160 by its HLA-C physiological ligand triggers a unique cytokine profile secretion in the cytotoxic peripheral blood NK cell subset. J. Immunol. 2004, 173, 5349-5354. [CrossRef]

15. Le Bouteiller, P.; Barakonyi, A.; Giustiniani, J.; Lenfant, F.; Marie-Cardine, A.; Aguerre-Girr, M.; Rabot, M.; Hilgert, I.; MamiChouaib, F.; Tabiasco, J.; et al. Engagement of CD160 receptor by HLA-C is a triggering mechanism used by circulating natural killer (NK) cells to mediate cytotoxicity. Proc. Natl. Acad. Sci. USA 2002, 99, 16963-16968. [CrossRef]

16. Tsujimura, K.; Obata, Y.; Matsudaira, Y.; Nishida, K.; Akatsuka, Y.; Ito, Y.; Demachi-Okamura, A.; Kuzushima, K.; Takahashi, T. Characterization of murine CD160+ CD8+ T lymphocytes. Immunol. Lett. 2006, 106, 48-56. [CrossRef]

17. Murayama, T.; Takegoshi, M.; Tanuma, J.; Eizuru, Y. Analysis of human cytomegalovirus UL144 variability in low-passage clinical isolates in Japan. Intervirology 2005, 48, 201-206. [CrossRef]

18. Tu, T.C.; Brown, N.K.; Kim, T.J.; Wroblewska, J.; Yang, X.; Guo, X.; Lee, S.H.; Kumar, V.; Lee, K.M.; Fu, Y.X. CD160 is essential for NK-mediated IFN-gamma production. J. Exp. Med. 2015, 212, 415-429. [CrossRef]

19. Tyanova, S.; Temu, T.; Cox, J. The MaxQuant computational platform for mass spectrometry-based shotgun proteomics. Nat. Protoc. 2016, 11, 2301-2319. [CrossRef]

20. Omasits, U.; Ahrens, C.H.; Muller, S.; Wollscheid, B. Protter: Interactive protein feature visualization and integration with experimental proteomic data. Bioinformatics 2014, 30, 884-886. [CrossRef]

21. Kabsch, W. Xds. Acta Cryst. D Biol. Cryst. 2010, 66 Pt 2, 125-132. [CrossRef] 
22. Liu, W.; Vigdorovich, V.; Zhan, C.; Patskovsky, Y.; Bonanno, J.B.; Nathenson, S.G.; Almo, S.C. Increased heterologous protein expression in drosophila S2 cells for massive production of immune ligands/receptors and structural analysis of human HVEM. Mol. Biotechnol. 2015, 57, 914-922. [CrossRef] [PubMed]

23. Matthews, B.W. Solvent content of protein crystals. J. Mol. Biol. 1968, 33, 491-497. [CrossRef]

24. Vagin, A.; Teplyakov, A. Molecular replacement with MOLREP. Acta Cryst. D Biol. Cryst. 2010, 66 Pt 1, 22-25. [CrossRef]

25. Collaborative Computational Project. The CCP4 suite: Programs for protein crystallography. Acta Cryst. D Biol. Cryst. 1994, 50 Pt 5, 760-763. [CrossRef] [PubMed]

26. Giustiniani, J.; Bensussan, A.; Marie-Cardine, A. Identification and characterization of a transmembrane isoform of CD160 (CD160-TM), a unique activating receptor selectively expressed upon human NK cell activation. J. Immunol. 2009, $182,63-71$. [CrossRef] [PubMed]

27. Henry, A.; Boulagnon-Rombi, C.; Menguy, T.; Giustiniani, J.; Garbar, C.; Mascaux, C.; Labrousse, M.; Milas, C.; Barbe, C.; Bensussan, A.; et al. CD160 expression in retinal vessels is associated with retinal neovascular diseases. Invest. Ophthalmol. Vis. Sci. 2018, 59, 2679-2686. [CrossRef]

28. Menguy, T.; Briaux, A.; Jeunesse, E.; Giustiniani, J.; Calcei, A.; Guyon, T.; Mizrahi, J.; Haegel, H.; Duong, V.; Soler, V.; et al. Anti-CD160, alone or in combination with Bevacizumab, is a potent inhibitor of ocular neovascularization in rabbit and monkey models. Invest. Ophthalmol. Vis. Sci. 2018, 59, 2687-2698. [CrossRef]

29. Cheung, T.C.; Steinberg, M.W.; Oborne, L.M.; Macauley, M.G.; Fukuyama, S.; Sanjo, H.; D'Souza, C.; Norris, P.S.; Pfeffer, K.; Murphy, K.M.; et al. Unconventional ligand activation of herpesvirus entry mediator signals cell survival. Proc. Natl. Acad. Sci. USA 2009, 106, 6244-6249. [CrossRef] [PubMed]

30. Proll, J.; Bensussan, A.; Goffin, F.; Foidart, J.M.; Berrebi, A.; Le Bouteiller, P. Tubal versus uterine placentation: Similar HLA-G expressing extravillous cytotrophoblast invasion but different maternal leukocyte recruitment. Tissue Antigens 2000, 56, 479-491. [CrossRef] [PubMed] 